\section{El compromiso social de la universidad, un reto "glocal"}

UTE. Revista de Ciències de l'Educació

Monogràfic 2018. Pàg. 83-99

ISSN 1135-1438. EISSN 2385-4731

http://revistes.publicacionsurv.cat/index.php/ute

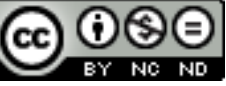

DOI: https://doi.org/10.17345/ute.2017.3.1941

\author{
Mònica Figueras Maz \\ Universitat Pompeu Fabra. Departamento de Comunicación
}

Silvia Ayuso Siart

Directora académica de la Cátedra MANGO de RSC

Rebut: 14/07/2017 Acceptat: 20/09/2017

\title{
Resumen
}

El objetivo del artículo es identificar y analizar la integración de la responsabilidad social en las principales funciones ejercidas por las universidades, considerando la doble vertiente global-local. Nos centraremos en la implantación de la responsabilidad social universitaria (RSU) en los ámbitos de la gestión institucional, formación académica, formación pedagógica e investigación, e ilustraremos cada ámbito mediante una iniciativa destacada de las universidades catalanas. Aunque de forma todavía incipiente y desarticulada, existen ya experiencias interesantes de cómo afrontar el reto "glocal" desde las distintas misiones de la universidad.

Palabras clave: responsabilidad social, compromiso social, universidad.

\begin{abstract}
The objective of this paper is to identify and analyze the integration of social responsibility in the main functions of universities, the global-local double. We will focus on the implementation of university social responsibility (RSU) in the areas of institutional management, academic training, pedagogical training and research, and we will illustrate each area through a prominent initiative of Catalan universities. Although still incipient and unorganized, there are already interesting experiences of how to face the "glocal" challenge from the different missions of the university.
\end{abstract}

KeyWords: social responsibility, social commitment, university social exclusion, inclusive education, story, educational proposal. 


\section{Introducción}

A diferencia de otras instituciones, el compromiso o responsabilidad social (RS) de las universidades está ya implícito por naturaleza en su propia finalidad. Tanto la primera misión de las universidades como la segunda, la docencia y la investigación, tienen que ver con la función de servicio y responsabilidad con la sociedad. La universidad genera su mayor impacto a través de la formación de los estudiantes que son ciudadanos y serán los futuros profesionales, líderes o dirigentes de la sociedad. La investigación, por su parte, busca el avance social a través de la aplicación de sus resultados. Pero además, y de forma a veces poco consciente, todas las universidades como instituciones han venido ejerciendo acciones y políticas orientadas a dar servicio a la sociedad más allá de esas dos misiones. Algunas lo han incorporado a la estrategia de la universidad, en otras, en cambio, es menos visible, pero lo cierto es que en los últimos años ha habido un avance en las universidades españolas con respecto al compromiso social mostrado, a través de diferentes programas de acción social, medidas de carácter medioambiental, iniciativas que promueven la igualdad y transparencia, etc.

La Estrategia Universidad 2015 se hizo eco acerca del nuevo rol que se esperaba de las universidades y del modo idóneo de materializarlo al tiempo que incorporaba las políticas europeas en materia de responsabilidad social de las organizaciones, investigación e innovación y enseñanza superior. Aparte de adoptar las recomendaciones emanadas de la implementación del Espacio Europeo de Educación Superior (EEES), la Estrategia Universidad 2015 se refiere a la responsabilidad social del sistema universitario y de las universidades que lo integran como un eje estratégico transversal que ha de ser tenido en cuenta en todas las misiones desempeñadas, así como en las capacidades, personas, gestión interna y relación con los entornos (Ministerio de Educación, 2011a). Esta Estrategia apuesta por una concepción amplia de la responsabilidad social universitaria (RSU) como elemento integral de la tercera misión. Más allá de las misiones relacionadas con la formación y la investigación, la tercera misión implica la transferencia de conocimiento y tecnología, pero también y en un sentido más general, el compartir conocimiento con la sociedad, el compromiso con la comunidad, la interacción con organismos de la sociedad civil, la contribución a la resolución de los grandes problemas sociales de nuestros días, la implicación con el desarrollo económico y social del entorno, etc.

Otra de las iniciativas desarrolladas por parte del Gobierno español fue la creación de la Comisión Técnica de la Estrategia Universidad 2015, que en 2011 elaboró un documento de trabajo que lleva por título "La responsabilidad social de la universidad y el desarrollo sostenible". El informe tenía por objetivo realizar un análisis y propuestas de actuación acerca del concepto de responsabilidad social de la universidad y de su relación con el desarrollo sostenible, una vez se había aprobado la Ley 2/2011 de Economía Sostenible (Ministerio de Educación, 2011b). Define la responsabilidad social del sistema universitario y de las universidades como "una reconceptualización del conjunto de la institución universitaria, a la luz de los valores, objetivos, formas de gestión e iniciativas que implican un mayor compromiso con la sociedad y con la contribución a un nuevo modelo de desarrollo, más equilibrado y sostenible" (pág. 33).

En el contexto internacional, la Estrategia Europa 2020, que establece las bases del crecimiento de la Unión Europea para los próximos años, pide que se desarrolle una economía inteligente, sostenible e integradora, que debería de contribuir a que se genere ocupación, productividad y cohesión social. Concretamente, los objetivos se refieren a cinco elementos esenciales: la ocupación, la innovación, la educación, la integración social y el clima y energía. Las universidades son también agentes clave en este nuevo escenario para abordar el doble reto de las sociedades actuales: construir sistemas económicos sostenibles y competitivos a escala global que, a la vez, puedan atender y dar respuesta adecuada a las problemáticas sociales crecientes. 
En este sentido, las universidades están reconociendo y poniendo de relevancia el papel que juegan en contribuir a la transformación social y el desarrollo sostenible a través de sus misiones. Este reconocimiento se hace a partir de la RSU, que puede definirse como el "conjunto de actuaciones que realiza la universidad como resultado de la ejecución de su proyecto institucional, donde declara explícitamente a través de su visión y misión, la orientación y coherencia de sus valores y actividades con la búsqueda de la felicidad de los seres humanos, y de la consolidación de una sociedad más justa y equilibrada con objetivos de largo plazo que permitan lograr las condiciones necesarias y suficientes para que nuestro planeta Tierra sea un hogar confortable para las futuras generaciones" (Martínez y Picco, 2001). En la misma línea que lo anterior, en el marco del proyecto "Universidad Construye País" (cit. en Larrán y Andrades, 2013), se define la RSU desde una perspectiva de los valores y principios que deben fundamentar la misión universitaria como "la capacidad que tiene la universidad de difundir y poner en práctica un conjunto de principios, valores generales y específicos, por medio de cuatro procesos claves: gestión, docencia, investigación y extensión universitaria, respondiendo así ante la propia comunidad universitaria y ante el país donde está inserta".

La RSU es una adaptación a las dinámicas de las instituciones de educación superior de la responsabilidad social corporativa (RSC) (Larrán y Andrades, 2013). En su implantación práctica, la RSU debería abordar cuatro ámbitos clave de toda institución universitaria: la gestión de la propia organización, la formación académica y pedagógica, la investigación, y la extensión universitaria (iGR, 2016; Ruiz-Corbella y Bautista-Cerro, 2016). Más allá de las acciones específicas de participación social que llevan a cabo (ACUP, 2011), las universidades se enfrentan al reto de materializar su compromiso social en actividad ordinaria, conciliando la perspectiva global y local (Grau, 2014). Por un lado, existe una tendencia hacia la definición de principios globales de RS, promovida por iniciativas como el Pacto Mundial de las Naciones Unidas y las directrices para elaborar informes de sostenibilidad, como la guía del Global Reporting Initiative (GRI). Por otro lado, los problemas a abordar por las políticas de RS son diferentes en cada contexto local, ya que están influidos por distintas variables institucionales, socioeconómicas y culturales.

El objetivo del artículo es identificar y analizar la integración de la responsabilidad social en las principales funciones ejercidas por las universidades, considerando la doble vertiente global-local. A continuación, nos centraremos en la implantación de la RSU en los ámbitos de la gestión institucional, formación académica, formación pedagógica e investigación, e ilustraremos cada ámbito mediante una iniciativa destacada de las universidades catalanas.

\section{La RSU en la gestión institucional}

En primer lugar, la integración de la RSU debe realizarse a nivel institucional. En la actualidad, numerosas universidades españolas han incluido la RSU en su organigrama (Ruiz-Corbella y Bautista-Cerro, 2016). Las formas que adoptan estos órganos que se ocupan específicamente de la RS son diversos, desde órganos de gobierno (vicerrectorados, comisionados) a unidades técnicas como oficinas o comisiones. En su estudio, Ruiz-Corbella y Bautista-Cerro (2016) observan que son escasas las universidades que cuentan con un Vicerrectorado que se ocupe explícitamente de la RSU, como, por ejemplo, la Universidad de Cádiz (Vicerrectorado de Responsabilidad Social Extensión Cultural y Servicios Universitarios), o la Universidad Carlos III (Vicerrectorado de Estudiantes, Responsabilidad Social e Igualdad), pero que prácticamente todas las universidades públicas cuentan con algún vicerrectorado que incluye, al menos en su denominación, aspectos que pueden englobarse en la RSU, como sostenibilidad, desarrollo sostenible, medio ambiente, cooperación internacional o igualdad. En muchos casos, también asumen competencias y funciones en RS determinadas unidades técnicas específicas, como pueden ser Oficinas Verdes, de Cooperación o de Igualdad. 
Por otro lado, un creciente número de universidades españolas presentan o han presentado una memoria de las actividades que desarrollan y que pueden ser enmarcadas en el área de la RS, bien sea con un formato institucional propio, siguiendo el modelo de informe del Pacto Mundial o siguiendo las recomendaciones formuladas por la GRI (Ruiz-Corbella y Bautista-Cerro, 2016). Por ejemplo, desde el curso 2008-2009 la Universitat de Barcelona elabora anualmente una memoria de responsabilidad social, en la que compila las principales magnitudes de su impacto sobre la sociedad de acuerdo con indicadores propuestos por la GRI e indicadores de elaboración propia. También existen casos de universidades que, sin desarrollar una memoria independiente de RSU, sí incluyen en las memorias académicas las actividades de RSU. Finalmente, otras universidades mantienen un espacio web con gran cantidad de información sin llegar a publicar memorias.

Pero, más allá de las memorias, para asegurar el apoyo y la implicación de todos los niveles organizativos de la universidad, se recomienda incorporar la RS de manera explícita en la declaración de la misión, visión y valores de la institución, así como en sus planes estratégicos (Setó-Pamies \& Papaoikonomou, 2016). Esto contribuirá a establecer una cultura orientada a la RS a largo plazo y con independencia del equipo directivo.

La integración de los principios de la RS en las universidades requiere la colaboración de diferentes actores (profesores, estudiantes, personal administrativo, etc.) y constituye un importante cambio organizativo. En este sentido, el proceso de integración en la gestión universitaria también debe contar con una planificación formal mediante la creación de infraestructuras -en términos de organización y presupuesto- que apoyen la iniciativa y resuelvan posibles problemas que surjan, como la falta de voluntad para cambiar debido a la carga de trabajo adicional, la actitud conservadora hacia el cambio y la falta de información sobre cómo se puede llevar a cabo el cambio (Lozano, 2006; Jabbour et al., 2013).

\subsection{Proyecto: El Plan Estratégico de la Universitat Pompeu Fabra}

La Universitat Pompeu Fabra (UPF), en los últimos años, ha concedido una mayor y creciente importancia a los temas relacionados con la Responsabilidad Social (RS). Esta toma de consciencia culminó en el año 2013 con la creación del vicerrectorado específico sobre esta temática. Previamente, en el 2012 se había creado el Programa de Responsabilidad Social, cuya coordinación se asignó al Gabinete del Rectorado de la universidad. Los retos que se planteaban con la creación de un vicerrectorado que se dedicara casi exclusivamente a los temas de responsabilidad social eran diversos: ¿Cómo conseguir introducir la RS en la cultura de toda la comunidad universitaria sin hacerlo desde la imposición? ¿Cómo hacer que orgánicamente la RS fuese incorporada en todos los objetivos de las unidades y servicios? ¿Cómo elaborar un plan director participativo de RS (no "sectorial") que implicase a todos los stakeholders (internos y externos)? ¿Cómo hacer que sobreviviera a los diferentes equipos de gobierno?

Coincidentemente, el equipo de gobierno de la universidad decidió iniciar la elaboración del Plan Estratégico de la UPF para el periodo 2015-2025. Un plan a largo plazo concebido para ser implantado mediante planes de actuación bianuales. En este marco, se abría una oportunidad para hacer confluir el nuevo proyecto estratégico con los retos en materia de responsabilidad social. Se consideró óptimo integrar los retos en RS de la educación superior en el proceso profesionalizado y participativo que se había diseñado para la elaboración del Plan Estratégico.

En la fase inicial de elaboración del Plan Estratégico se hizo una tarea de benchmarking para buscar planes estratégicos de otras universidades -de relevancia nacional e internacional- que 
pudieran servir como referentes tanto a nivel de metodología como de contenido. En este contexto, y para garantizar que el nuevo plan fuese inclusivo y representara a toda la comunidad universitaria, se consideró indispensable iniciar un proceso participativo donde pudieran colaborar todos los colectivos que integran la UPF (PAS, PDI, alumnos, exalumnos, stakeholders, partners...). Así, se definieron cinco ámbitos temáticos específicos a la par que se crearon dos ejes transversales para trabajar (tanto en el diagnóstico como en las propuestas) dos ámbitos estratégicos que están presentes en cada uno de los otros cinco: la internacionalización y el compromiso (véase Figura 1).

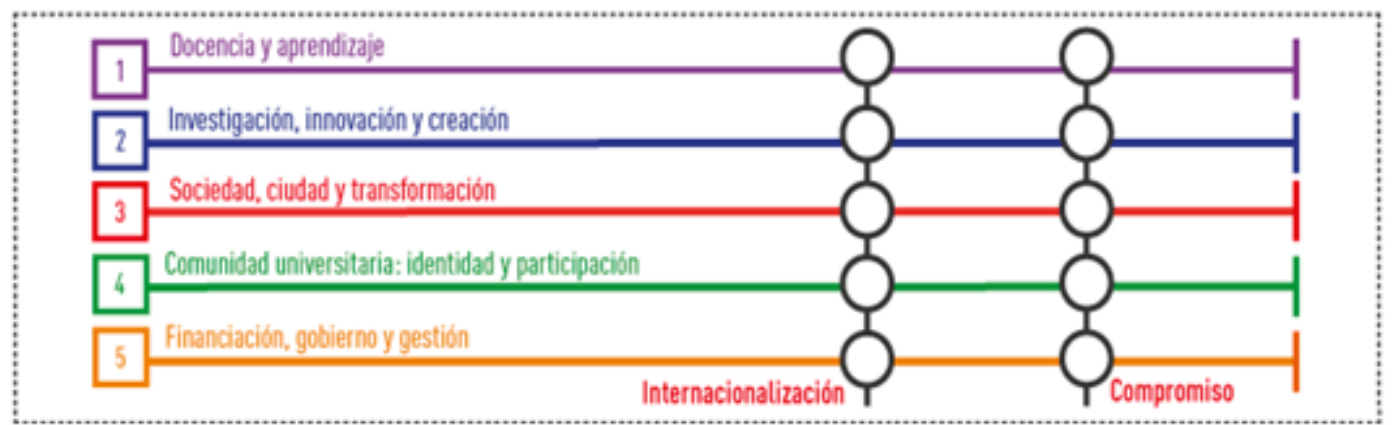

Figura 1. Grupos de trabajo para la elaboración del Plan Estratégico UPF 2015-2025.

Se crearon grupos de trabajo específicos por ámbito formados por una veintena de personas cada uno que desarrollaron el diagnóstico y las propuestas durante unas cinco sesiones de trabajo. Éstos estaban compuestos por un coordinador y representantes de cada uno de los grupos que integran la comunidad universitaria. Además, para complementar la pluralidad de perspectivas, se incluyeron miembros del Consejo Social de la universidad, Alumni y expertos externos a la institución. Fue clave incluir en estos grupos persona "afines" a la RS que velaran porque estos temas afloraran en las distintas sesiones. Desde el vicerrectorado de Responsabilidad Social se elaboró un informe para dar a conocer la RSU y, en concreto, datos cualitativos sobre la evolución y la situación actual de la RSU en la UPF, así como retos y propuestas sobre líneas de actuación en el futuro. El informe se articuló a partir de cuatro ejes de actuación: docencia, investigación, políticas institucionales y la relación con la sociedad. Este informe sirvió de referencia a los miembros de los diferentes grupos de trabajo para definir propuestas de RSU en relación con su ámbito estratégico. La propia vicerrectora de RSU o expertos externos en RS dinamizaron una sesión de trabajo en cada uno de los grupos.

Además, se realizaron otras acciones participativas específicas como la selección de buenas prácticas de otras universidades y la activación de mecanismos de participación online donde la comunidad podía participar votando y opinando sobre si esas acciones eran interesantes para la propia universidad; la organización de una mesa redonda temática de RS con expertos para dar a conocer esa dimensión hasta entonces no muy conocida; o la realización de grupos de discusión con cada uno de los colectivos y de foros de debate presenciales.

El Plan Estratégico 2015-2025 se presentó en febrero de 2017 coincidiendo con los actos de celebración del vigesimoquinto aniversario de la Universitat Pompeu Fabra. El proceso de elaboración de este documento de planificación estratégica y todas las actividades asociadas están teniendo un impacto evidente en toda la comunidad universitaria y, en concreto, en lo que a la RSU se refiere. Un proceso de este tipo permite que los miembros de toda la comunidad universitaria se vean abocados a una tarea de evaluación y de conocimiento de la evolución y el estado actual de la institución. Además, también sirve de punto de encuentro e intercambio de concepciones entre los miembros de los diferentes colectivos. 
Pero, de especial modo, cabe recalcar la contribución del proceso de elaboración del nuevo plan en la difusión de las ideas centrales de la RS en todos los sectores de la universidad, y de la importancia de impregnar de la "cultura" necesaria a los miembros de la institución. También ha servido para tomar consciencia de las acciones ya existentes en materia de RS y, en algunos casos, de la contribución a ella por parte de muchos miembros de la comunidad. Además, se valora positivamente, por un lado, la integración de forma natural de la RS en el Plan Estratégico, mediante la creación de un eje temático transversal dedicado al compromiso (evitando así crear algo "sectorial"); $y$, por otro lado, la oportunidad que representa el nuevo plan de convertir la RS en una estrategia no solo del equipo de gobierno, sino también aspirada por todos los miembros de la comunidad. En definitiva, haber desarrollado un proyecto de este tipo ha supuesto una oportunidad para avanzar en la implantación de la Responsabilidad Social y sentar las bases para su futuro en la Universitat Pompeu Fabra.

\section{La RSU en la formación académica}

Con la creciente toma de conciencia de los problemas de la sostenibilidad que afronta la humanidad y el planeta, desde finales del siglo XX cada vez más universidades han manifestado su voluntad de introducir el desarrollo sostenible en la formación que ofrecen, como muestran la Declaración de Talloires (1990) o la Carta Copernicus (1993). En el año 2002 las Naciones Unidas proclamaron, para el período 2005-2014, el Decenio de la Educación para el Desarrollo Sostenible, designando a la UNESCO como organismo ejecutor del proyecto. Este programa hacía hincapié en el papel crucial de la educación y de la formación para la consecución de una comunidad mundial más sostenible, siendo su objetivo central la integración de los valores, actividades y principios vinculados intrínsecamente al desarrollo sostenible en todas las formas de la educación y el aprendizaje. La Declaración Bucarest (2012) sobre el aprendizaje centrado en el estudiante, hace referencia a los métodos innovadores que propicien el pensamiento crítico, el desarrollo sostenible y la responsabilidad social y la Conferencia de Naciones Unidas sobre el Desarrollo Sostenible en 2012 (Río+20) se refiere explícitamente al compromiso de prácticas de sostenibilidad en instituciones de educación superior.

A la hora de integrar el contenido de la RS en los planes de estudio académicos se pueden seguir diferentes estrategias, en función de las decisiones que se tomen con respecto a algunos aspectos clave (Setó-Pamies \& Papaoikonomou, 2016). Una de las principales cuestiones a tener en cuenta es si el contenido de RS debe estar concentrado o disperso en el currículum universitario. En el primer caso, se trata de diseñar asignaturas específicas que se centren en temas relacionados con la sostenibilidad, la ética o la RSC. En el segundo caso, se considera necesaria la formación transversal en RS y se incorpora mediante contenidos específicos en diferentes asignaturas pertenecientes a diferentes áreas y niveles. La incorporación de contenidos de RS a través de asignaturas específicas (stand-alone subjects) o integrado en otras asignaturas (embedded subjects) sigue siendo un debate abierto en la actualidad (Christensen et al., 2007; Hartman y Werhane, 2009). Por un lado, las asignaturas específicas permiten profundizar en las relaciones entre la RS y cada disciplina. La principal ventaja es que otorga a la RS una identidad claramente definida dentro de la disciplina, al mismo nivel que otras asignaturas. La mayor desventaja, sin embargo, es que la RS corre el riesgo de ser vista como una cuestión separada, desconectada de otras cuestiones teóricas y prácticas de la titulación. Por otro lado, la integración en otras asignaturas posibilita a los docentes desafiar las visiones del mundo de los estudiantes y animarlos a analizar explícitamente sus supuestos sobre la actividad humana, el medio ambiente y la sociedad. La ventaja de esta opción es que la RS está integrada en todo o gran parte del plan de estudios de una titulación y tiene un impacto en un mayor número de estudiantes. No obstante, 
su principal desventaja es que requiere un compromiso de toda la universidad o facultad acerca del tiempo, los recursos y el apoyo.

Con respecto a las asignaturas específicas de RS, una decisión que se debe tomar es si estas asignaturas deben ser obligatorias $u$ optativas. Cuando se incorporan asignaturas específicas obligatorias en el currículum universitario, la RS se convierte en un elemento no negociable del plan de estudios para el estudiante. Pero cuando estos mismos cursos son optativos es el estudiante quien decide elegirlos en última instancia. Por un lado, los cursos obligatorios de RS llegan a todos los estudiantes. Por otra parte, los cursos optativos sobre estos temas implican que ciertos estudiantes no reciban ningún tipo de educación relevante en RS debido a la falta de interés o al coste de oportunidad (otros cursos optativos son más atractivos). Por lo tanto, la inclusión de asignaturas obligatorias en RS puede indicar que la RS es vista como una competencia básica para los estudiantes universitarios. Otra cuestión importante a considerar es si la asignatura debe centrarse en una sola disciplina o adoptar un enfoque multidisciplinar. Dada la naturaleza de la RS y del desarrollo sostenible, varios autores sostienen que se requiere una perspectiva interdisciplinaria, multidisciplinaria o más bien transdisciplinaria, porque el conocimiento de diferentes disciplinas puede permitir una comprensión más holística de la sostenibilidad (Lozano et al., 2014; Sibbel, 2009).

En el ámbito universitario español ha habido un creciente interés por integrar la responsabilidad social y la sostenibilidad en el currículum. La Comisión Sectorial de Sostenibilidad de la Conferencia de Rectores de las Universidades Españolas (CRUE) elaboró en 2005 las "Directrices para la sostenibilización curricular", que se revisaron en 2011 (CRUE, 2012). En este documento se hace mención a los aspectos que deben considerarse para implementar en los currículos contenidos en sostenibilidad, para fomentar un cambio de actitudes en los futuros profesionales de manera que comprendan cómo su trabajo interactúa local y globalmente con la sociedad, por medio de la aplicación de un enfoque holístico a los problemas socio-ambientales. En consecuencia, las universidades españolas han incorporado asignaturas relacionadas con la RS y la sostenibilidad en los planes de estudio de grados y posgrados, sobre todo en las áreas de economía, finanzas, empresa, ingeniería, ciencias ambientales y turismo (Ruiz-Corbella y BautistaCerro, 2016).

\subsection{Proyecto: Educación para el desarrollo sostenible en la universidad}

El proyecto "Education for Sustainable Development at University" (ESDU) es una iniciativa orientada a la promoción específica y transversal de la Educación para el Desarrollo (ED) en el ámbito formal universitario, con el objetivo de potenciar el papel de la universidad en la generación de conocimiento crítico y en la formación de personas que incorporen una mirada ética en el ejercicio de su futura profesión, a la vez que se convierten en parte de una ciudadanía activa y comprometida con un desarrollo sostenible desde una doble perspectiva local - global (Peix et al., 2017).

En marcha desde el curso 2013-2014, el proyecto ESDU está liderado por la Fundació Autònoma Solidària (FAS), en colaboración con la Asociación Catalana de Universidades Públicas (ACUP), y se articula mediante una estrategia de trabajo en red que agrupa centros y facultades, profesorado, estudiantes, oficinas de cooperación universitaria y entidades con experiencia en ED, entre otros, con el apoyo del Ayuntamiento de Barcelona.

Se estructura en dos grandes áreas de intervención (véase Figura 2). La primera se ubica en la Universitat Autònoma de Barcelona (UAB) y está orientada a estimular la incorporación de 
contenidos, habilidades y valores con pauta de ED en los estudios de grado, a través de acciones de incidencia en la formación del alumnado y de fortalecimiento de la calidad y la innovación docente. La segunda implica al conjunto de las universidades públicas catalanas y apuesta por la creación de espacios de trabajo colaborativo entre agentes universitarios y sociales que promuevan el diseño de acciones para la implantación de este enfoque y, particularmente, de los Objetivos de Desarrollo Sostenible (ODS) ${ }^{1}$ en la docencia universitaria reglada.

En el área de trabajo de la incidencia curricular en la UAB contempla las siguientes acciones de formación del alumnado, de fortalecimiento de las capacidades docentes y de facilitación de recursos pedagógicos:

- Talleres sobre los Objetivos de Desarrollo Sostenible (ODS): Se trata de una propuesta formativa que consta de talleres teórico-prácticos en el aula y de actividades complementarias diseñadas de forma colaborativa con el profesorado para introducir la Agenda 2030 para el Desarrollo Sostenible en la formación inicial de estudiantes de diferentes disciplinas. A lo largo del curso 2016-2017 se ha programado una prueba piloto que implica a la Facultad de Ciencias de la Educación y a la Escuela de Ingeniería de la $U A B$, con la voluntad de evaluar y revisar esta primera experiencia y poder extenderla a otros centros del mismo campus durante los cursos venideros.

- Grupos de Educación para el Desarrollo en las Facultades (GEDEF): Se trata de grupos de trabajo horizontal formados por miembros del decanato, profesorado, estudiantes y organizaciones sociales, en los que se plantean propuestas de incorporación de la ED en los estudios de grado. Actualmente, se encuentran en activo en las facultades de Veterinaria, Economía y Empresa y en la Escuela de Ingeniería para incentivar el tratamiento académico de temáticas como la soberanía alimentaria, la economía social y solidaria y el desarrollo humano sostenible, respectivamente en los distintos centros.

- Minor de Desarrollo Sostenible y Ciudadanía Global: De carácter transversal e interdisciplinar, es un itinerario universitario dirigido al alumnado de grado de cualquier titulación de la UAB para cursar como créditos optativos. Coordinado por la Facultad de Ciencias Políticas y Sociología, su objetivo es proporcionar una formación básica en estudios sociales desde la perspectiva del desarrollo sostenible y bajo la concepción de una ciudadanía global que acerque al alumnado a las aproximaciones científicas en este ámbito. El plan de estudios del minor consta de 60 ECTS, de los cuales el estudiante cursa un mínimo de 30, y está constituido por asignaturas de siete facultades y ocho grados diferentes (Sociología, Periodismo, Economía, Educación Social, Antropología, Geografía, Derecho y Ciencias Ambientales). Se integró de forma permanente en la oferta formativa de la UAB en el curso 2014-15.

- Formación dirigida al profesorado universitario: La formación de profesorado constituye una de las principales líneas de trabajo del proyecto y se lleva a cabo con la colaboración de la Oficina de Calidad Docente (OQD) de la UAB, hecho que permite un reconocimiento en el currículum de formación docente. Por un lado, se han creado e incorporado al plan de formación permanente dos cursos orientados a facilitar herramientas para el desarrollo de las competencias del pensamiento crítico y el compromiso ético en la tarea docente. Por otro lado, hay actividades de formación a medida vinculadas a los espacios de colaboración con la Escuela de Ingeniería y la Facultad de Veterinaria que se centran en cuestiones del ámbito del desarrollo humano sostenible y la soberanía alimentaria, respectivamente.

- Banco de recursos dirigido a la educación superior: Colección de referencia en propuestas pedagógicas y recursos sobre diferentes temáticas de ED, para aplicar en el aula

\footnotetext{
${ }^{1}$ En el apartado sobre investigación se describe en detalle el origen y alcance de los ODS.
} 
universitaria integrada a la nueva red de recursos de educación transformadora promovida por la entidad EduAlter.

En el ámbito del trabajo en red con universidades se busca la generación de nuevas sinergias y de espacios de trabajo con actores universitarios y sociales que fomenten la discusión y el diseño de estrategias para la integración de un enfoque de ED en las universidades públicas catalanas. Además, se intentan forjar relaciones en este campo también a nivel estatal e internacional.

Una de las primeras colaboraciones en el afán de tejer redes con las universidades catalanas fue la elaboración del informe "La ED en la universidad. Impulsando la educación crítica y comprometida en los estudios de grado" (Villà, 2015), una diagnosis sobre el estado actual de implantación, las posibilidades y estrategias en las universidades públicas catalanas. De forma paralela se han organizado una serie de seminarios de trabajo, que pretenden ser un espacio de intercambio y construcción colectiva entre profesorado, oficinas de cooperación, entidades, y organismos con capacidad de incidencia en la materia como la misma ACUP, la Agencia para la Calidad del sistema Universitario de Cataluña (AQU) o LaFede.Cat, la red para la justicia global que agrupa más de un centenar de organizaciones sociales. A partir también de los resultados de estos espacios y con el fin de dar respuesta a las necesidades de incidencia política y de coordinación institucional detectadas, se ha impulsado la conformación de un grupo de trabajo interuniversitario en Educación para el Desarrollo Sostenible adscrito a la Comisión de Responsabilidad Social Universitaria de la ACUP. Cuenta con la participación de vicerrectorados y de personal técnico de las ocho universidades públicas catalanas $y$, por tanto, de representantes directos en los órganos de decisión política universitarios. El grupo, que se constituyó en febrero de 2016, ha recogido las propuestas trazadas en los seminarios y ha establecido su misión y plan de trabajo en función de las líneas previamente consensuadas, con el único objetivo de fomentar la institucionalización de una educación superior con enfoque de desarrollo sostenible.

Este proceso emprendido a nivel catalán cumplió en septiembre de 2017 con la adopción de una declaración por parte de las ocho universidades públicas catalanas que implica un compromiso formal en materia de Objetivos de Desarrollo Sostenible. Desde los espacios de trabajo en red se facilitará una batería de propuestas que orienten y favorezcan la implementación efectiva de la declaración en las distintas universidades. 


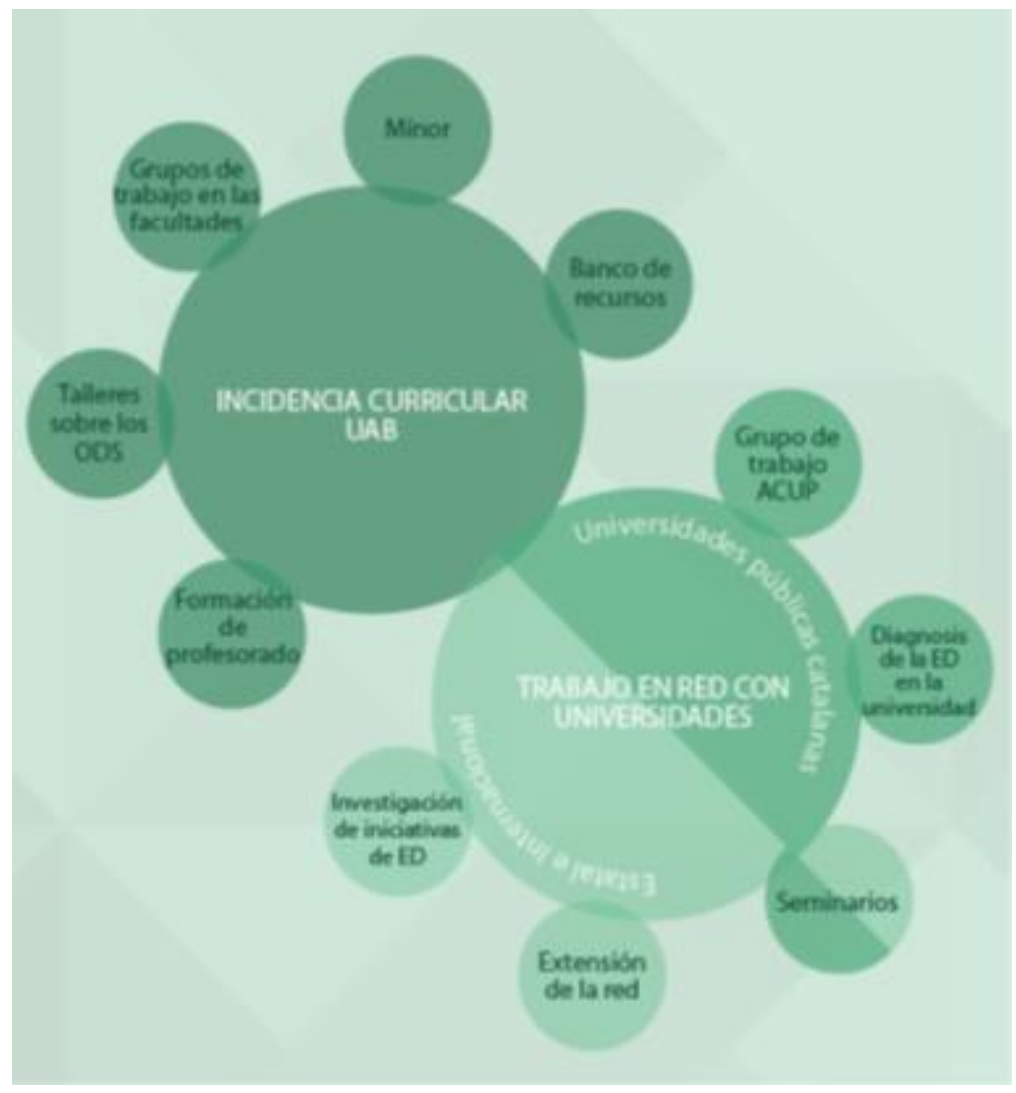

Figura 2: Áreas de intervención del proyecto ESDU.

Fuente: Fundació Autònoma Solidària (http://www.uab.cat/web/fas)

\section{La RSU en la formación pedagógica}

La integración de la RS en la enseñanza universitaria debe abordar tanto el ámbito de contenidos como el de procedimientos metodológicos (Ryan and Cotton, 2013). En este sentido, se suele asociar la docencia en RS con la innovación en los enfoques pedagógicos para que los estudiantes puedan desarrollar las competencias transversales para la sostenibilidad propuestas por la CRUE, entendidas como el "conjunto complejo e integrado de conocimientos, destrezas, habilidades, actitudes y valores que capacitan para operar y transformar la realidad con criterios de sostenibilidad" (CRUE, 2012). La literatura sobre educación para el desarrollo sostenible reclama innovaciones pedagógicas que proporcionen un aprendizaje interactivo, experiencial, transformador y apegado al mundo real (Rowe, 2007; Sipos et al., 2008; Steinemann, 2003).

Algunas de las estrategias didácticas más acordes con los principios de la sostenibilidad son las dialógicas (discusión, debate, diálogo); la reflexión crítica sobre temas relevantes a nivel local y global; casos hipotéticos y reales (role play y estudio de casos); colaboración en proyectos y tareas (actividades en grupo); actividades de aprendizaje experiencial como la acción comunitaria y la resolución de problemas; mentorías (para facilitar la comprensión y el compromiso con los temas de la educación para el desarrollo sostenible); exploración y resolución de problemas interdisciplinares; aprendizaje en la acción (en el que los aprendices desarrollan e implementan planes de acción, reflexionan sobre su experiencia y sugieren mejoras); y la investigación-acción (proceso cíclico de planificación, acción, observación y reflexión basado en la investigación para innovar y mejorar la práctica (Aramburuzabala et al., 2015). En todas estas metodologías, la característica común es que los estudiantes están involucrados en el proceso de aprendizaje y se les anima a desarrollar habilidades de pensamiento crítico y resolución de problemas. 
Desde esta perspectiva, el aprendizaje-servicio (ApS) se presenta como una de las metodologías idóneas para el desarrollo de competencias en RS y sostenibilidad. El ApS es una propuesta educativa que combina procesos de aprendizaje y servicio solidario a la comunidad en un solo proyecto, en el cual los participantes se forman trabajando sobre necesidades reales del entorno con el objetivo de mejorarlo (Furco \& Billing, 2002). Desde la óptica educativa, el ApS es un método de enseñanza-aprendizaje innovador y de carácter experiencial que permite dotar de más significado social a los aprendizajes académicos y formar en la responsabilidad social a los estudiantes universitarios (Martínez, 2010).

Por lo tanto, el ApS es una estrategia valiosa para la inclusión de la sostenibilidad en los curricula universitarios. Su enfoque es afín al concepto de Responsabilidad Social Universitaria contenido en el marco de la Estrategia Universidad 2015 y a las directrices aprobadas por la CRUE en 2005 y ratificadas en 2011 para la incorporación en todas las titulaciones universitarias de competencias transversales para la sostenibilidad. Así mismo, responde a la normativa universitaria actual, que reclama que las universidades favorezcan prácticas de responsabilidad social y ciudadana que combinen aprendizajes académicos en las diferentes titulaciones con la prestación de servicios a la comunidad, orientados a la mejora de la calidad de vida y la inclusión social (Marquès, 2014).

En este sentido, el Grupo de Trabajo de Sostenibilización Curricular de la CRUE propone la institucionalización del ApS en las universidades españolas para impulsar la sostenibilización curricular, contribuir al desarrollo de una sociedad más justa y mejorar los aprendizajes académicos y sociales que favorecen el desarrollo competencial de los estudiantes (CRUE, 2015). Esta metodología puede utilizarse en todas las titulaciones de grado y posgrado, integrarse en las programaciones docentes que aplica el profesorado en sus clases, en el marco de las prácticas externas de los estudiantes, e incorporarse en las ofertas temáticas abordadas en los trabajos fin de grado (TFG) y los trabajos fin de máster (TFM). Todo el profesorado formado previamente en ApS puede tutelar estos proyectos, que han de estar relacionados con el contenido de las asignaturas que imparten y aplicarse en organizaciones sociales y en instituciones educativas (asociaciones, ONG, fundaciones, instituciones públicas, etc.). Las acciones de servicio corresponden con las diferentes dimensiones de la sostenibilidad (social, económico y ambiental) y pueden centrarse en ámbitos tales como el medio ambiente, promoción de la salud, cooperación internacional, ayuda próxima a personas y colectivos con necesidades, apoyo en la educación, diversidad funcional, ciudadanía, patrimonio, etc.

En el contexto universitario catalán y estatal se percibe un creciente interés por el ApS universitario, que se manifiesta en la creación de redes e iniciativas que tienen por finalidad la promoción del ApS en las universidades, el intercambio de experiencias o la investigación en el ámbito de la innovación docente. En este sentido, cabe destacar la Red Española de AprendizajeServicio, creada el 2010 como espacio de colaboración entre instituciones educativas, organizaciones de acción social y profesionales que en diferentes ámbitos y Comunidades Autónomas impulsan desde hace años el ApS. En Cataluña, existen la Red APS(U), impulsada por el ICE de la Universitat de Barcelona que, entre otras actividades, organiza desde 2010 con carácter anual un encuentro sobre el ApS universitario, y el Centro Promotor de Aprendizaje Servició, que tiene como finalidad promover el estudio, la difusión y el desarrollo del ApS en todos los niveles educativos.

\subsection{Proyecto: Institucionalización del aprendizaje-servicio en la Universitat Rovira i Virgili}

La Universitat Rovira i Virgili (URV) ha sido pionera entre las universidades españolas en aprobar un programa de aprendizaje-servicio, cuyo objetivo es la plena institucionalización y extensión de esta metodología a todos los centros y titulaciones de la universidad, siguiendo para ello 
estándares y referentes internacionales tanto en relación con los elementos de calidad del ApS como sobre lo que implica un proceso de institucionalización en esta materia (Marqués, 2014).

Así, el Consejo de Gobierno de la URV aprobó el 23 de febrero de 2012 el Documento Marco del Programa de Aprendizaje Servicio. Este documento establece las bases de la institucionalización del ApS, metodología que ya contaba en la universidad con experiencias conocidas y reconocidas, fruto de la iniciativa individual o colectiva de un grupo de profesores y profesoras de distintos ámbitos de conocimiento. El 26 de febrero de 2015, se aprobó por el Consejo de Gobierno la actualización del Plan de Actuación para el periodo 2015-2017.

La institucionalización del ApS en la URV partió de un marco institucional favorable a la misma, en tanto que el Plan Estratégico Docente establece una competencia nuclear "C5. Comprometerse con ética y responsabilidad social como ciudadano/a y como profesional" y el Plan Estratégico de la Tercera Misión contempla de forma expresa el fomento del ApS y de la investigación basada en la comunidad como actuación asociada a los objetivos del eje 2 (social, de voluntariado y de cooperación al desarrollo).

Para la definición del Documento Marco del Programa de Aprendizaje Servicio en la URV, se tomaron por un lado como referencia las contribuciones teóricas de Andrew Furco (Furco, 2009) así como los conocidos estándares de calidad del ApS definidos por la Universidad de Stanford en 1998; y por otro lado en octubre de 2011 se visitaron in situ los programas de aprendizajeservicio de las siguientes universidades canadienses: University of Ottawa, University of Toronto, University of Guelph y Wilfrid Laurier University, gracias a una ayuda concedida por el Consejo Social de la URV.

La principal innovación de la iniciativa de la URV reside en el plan de actuación que incorpora y que se estructura en torno a los elementos de la institucionalización del ApS definidos por Furco, estableciendo para cada uno de ellos actuaciones asociadas, sus responsables, la temporización prevista y los recursos necesarios. Con alguna mínima adaptación con relación a la fuente original, dichos elementos de la institucionalitzación del ApS son:

- Definición y objetivos claros del ApS.

- Visión a largo plazo del rol del ApS en la institución.

- Vinculación del ApS a la misión institucional.

- Consideración del ApS como vehículo para alcanzar otros objetivos de la institución.

- Implicación y apoyo firme por parte del profesorado.

- Consideración del ApS como verdadera actividad académica del profesorado.

- Conocimiento por parte de los estudiantes de las opciones de ApS en la universidad.

- Rol activo de los estudiantes en la promoción del ApS en sus titulaciones.

- Consideración de los miembros de las entidades participantes como miembros de la comunidad universitaria.

- Existencia de una estructura de coordinación de las actividades ApS.

- Existencia de una estructura política para la promoción y supervisión del ApS.

- Existencia de personal adecuado y apropiado para la coordinación del ApS.

- Financiación suficiente para la coordinación, participación y promoción del ApS.

- Reconocimiento del ApS por parte de la institución.

- Existencia de un programa de seguimiento de la calidad del programa que permita evaluar las mejoras.

- Consideración del ApS por parte de Facultades y Escuelas como un componente valioso de sus titulaciones. 
En total han sido más de cuarenta actuaciones que han permitido en un plazo de dos años y con la ayuda inicial de una subvención del Ministerio de Educación, iniciar el proceso de institucionalización del ApS en la URV (elaboración de guías y materiales, implantación del procedimiento de validación de experiencias y de seguimiento de la calidad, diseño y ejecución de la estrategia de difusión, web del Programa, etc.) y posibilitar su lanzamiento a la comunidad universitaria y su difusión social.

El Programa de Aprendizaje Servicio de la URV cuenta ya con experiencias validadas y reconocidas, tanto en titulaciones de grado como de máster, en todos los ámbitos de conocimiento de la URV, y cuenta con 11 centros implicados. Desde que el Programa de ApS de la URV se aprobó en el curso académico 2012, y hasta el curso 2015-16 han participado 2.525 estudiantes y se han establecido colaboraciones con 346 entidades, instituciones locales e internacionales de diferentes tipologías (URV, 2017).

\section{La RSU en la investigación}

En la actualidad, como decíamos, las universidades se enfrentan a una doble responsabilidad a nivel local y global: por una parte, contribuir al desarrollo social, cultural y económico de sus regiones y países, y por otro lado, contribuir a los desafíos globales de la humanidad y el planeta, resumidos en los Objetivos de Desarrollo Sostenible (ODS) de las Naciones Unidas (GUNi, 2017). Estos diecisiete objetivos responden a cinco grandes ámbitos (planeta, prosperidad, personas, paz y partenariado) y se concretan en 169 hitos que cubren un amplio abanico de temáticas estratégicas a escala global, estatal, subestatal y local. Los ODS pretenden promover y fortalecer los derechos humanos, sociales y medioambientales y contribuir a resolver retos locales y globales y deberían ocupar la atención de las agendas de gobiernos, agentes sociales y empresas, y como no de las universidades y de la sociedad en general.

El conocimiento científico permite el progreso de la sociedad y es indispensable para su desarrollo. Los temas y enfoques de la investigación son clave para ver qué priorizan gobiernos, instituciones públicas y privadas y qué repercusiones pueden suponer local y globalmente. Contribuir a la transformación de la sociedad a través de los ODS incumbe fundamentalmente a la investigación. Investigación en el ámbito de la sostenibilidad ambiental pero también de la social y económica. La investigación sobre la Agenda 2030 y los ODS está cada vez más en la agenda tanto de instituciones internacionales que promueven la investigación como de organizaciones intergubernamentales y gubernamentales, $y$, obviamente, de las propias de las universidades.

Las universidades públicas catalanas son líderes en producción científica en el conjunto de las universidades españolas; en 2015 experimentaron una mejora considerable por lo que respecta a los fondos recibidos que se relaciona principalmente con un destacado progreso en la captación de fondos europeos (ACUP, 2017, pág.:116). De entre esta investigación, una parte empieza ya a converger con los ODS y afronta el reto global-local. En el último informe sobre la investigación en las universidades públicas catalanas se incluye un monográfico sobre su aportación en relación a los ODS para poner de relieve su contribución a encontrar soluciones a los retos planteados y analiza más de una veintena de ejemplos de las diferentes universidades (ACUP, 2017, pág.: 50114).

En el mismo sentido, ha aparecido con fuerza el concepto de "investigación e innovación responsables", la primera vez en $6^{\circ}$ Programa Marco de la UE (2002). En 2011 la Dirección General de Investigación en Bruselas publicó "A report on Responsible Research \& Innovation" con una primera aproximación a este concepto de RRI (por sus siglas en inglés) (Sutcliffe, 2011); más tarde introduce este concepto en su programa"Science with and for Society", con la explicitación de la 
participación ciudadana. La Declaración de Roma sobre Investigación e Innovación Responsables en Europa del año 2014 incorpora también este concepto. El objetivo es en todos los casos crear una sociedad donde las prácticas de investigación e innovación estén orientadas a conseguir resultados sostenibles, éticamente aceptables y socialmente deseables y relacionados con los valores, las necesidades y las expectativas de la sociedad. El programa marco de la Unión Europea para la investigación y la innovación Horizon 2020 (H2020) ha supuesto un estímulo para afrontar los retos global-local. H2020 plantea que la investigación y la innovación responsables son un enfoque que anticipa y evalúa las implicaciones potenciales y las expectativas de la sociedad con respecto a la investigación y la innovación, con el fin de fomentar el diseño de una investigación e innovación inclusivas y sostenibles. La RRI implica que actores sociales (investigadores, ciudadanos, políticos, empresarios, organizaciones del tercer sector, etc.) trabajen juntos durante todo el proceso de investigación e innovación para alinear mejor el proceso y sus resultados con los valores, necesidades y expectativas de la sociedad. En la práctica, la RRI se implementa como un paquete que incluye el compromiso de múltiples actores y público en la investigación y la innovación, permitiendo un acceso más fácil a los resultados científicos, la perspectiva de género, la ética en el contenido y el proceso de investigación y la educación científica formal e informal. La introducción de esta dimensión en las convocatorias competitivas europeas, y ahora también en las nacionales, está suponiendo un estímulo para que la investigación contribuya a avanzar en el desarrollo de las sociedades desde la perspectiva global-local o "glocal".

\section{Conclusiones}

En España, la Estrategia Universidad 2015 supuso una apuesta por una concepción amplia de la responsabilidad social universitaria (RSU) como elemento integral de la tercera misión. Más allá de la formación y la investigación, la tercera misión implica la transferencia de conocimiento y tecnología, pero también y, en un sentido más general, el compartir ese conocimiento con la sociedad, el compromiso con la comunidad y la interacción la sociedad civil.

La RSU o el compromiso social de las universidades debe traducirse no solo a través de acciones complementarias o paralelas a sus dos misiones sino también desde ellas de forma transversal. La universidades deben contribuir a la resolución de los grandes problemas sociales de la humanidad y el planeta e implicarse en el desarrollo económico y social del entorno. El reto global-local debe afrontarse también desde las instituciones de educación superior. Hay suficientes evidencias, tanto desde la literatura científica como de experiencias ya implementadas, para saber cómo hacerlo. Solo es necesaria una voluntad explícita.

A nivel institucional las universidades pueden integrar principios globales de desarrollo sostenible en el organigrama, las memorias y, sobre todo, la planificación estratégica a través de procesos participativos continuos con stakeholders. A nivel formativo, pueden integrar los desafíos globales en los contenidos curriculares, ya sea de forma concentrada a través de asignaturas o dispersa en el plan de estudios, y trasladar sus aportaciones a casos locales para fomentar un cambio de actitudes en los futuros profesionales y promover un aprendizaje experiencial. Finalmente, a nivel de la investigación, es posible introducir los ODS como objetos de estudio y la RRI como abordaje holístico, que integra agentes de la sociedad civil en el proceso y vela por transferir los resultados a las necesidades reales. Incorporar el compromiso social o la RS no es solo un desideratum, es una necesidad y hay atisbos de esperanza para el cambio. Aunque de forma todavía incipiente y desarticuladas, existen ya experiencias interesantes. En este artículo hemos visto ejemplos concretos en cada nivel de cómo afrontar el reto "glocal" desde las distintas misiones de la universidad. Queremos creer que en un futuro cercano no se trate de casos puntuales sino de una verdadera institucionalización de este compromiso en las universidades. 


\section{Referencias Bibliográficas}

ACUP - Associació Catalana d'Universitats Públiques (2011). Impactes de les universitats publiques catalanes a la societat, ACUP, Barcelona, http://www.acup.cat/sites/default/files/impactes-de-lesuniversitats-publiques-catalanes-la-societat_0.pdf

ACUP - Associació Catalana d'Universitats Públiques (2017) Indicadors de recerca i innovació de les universitats públiques catalanes 2016. http://www.indicadorsuniversitats.cat/recerca/documents/2016/informe_2016.pdf

Aramburuzabala, P., Cerrillo, R. \& Tello, I. (2015) Aprendizaje-servicio: una propuesta metodológica para la introducción de la sostenibilidad curricular en la universidad. Profesorado, 19(1): 78-95.

Christensen, L.J., Pierce, E., Hartman, L.P., Hoffman, W.H. \& Carrier, J. (2007) Ethics, CSR, and sustainability education in the Financial Times top 50 global business schools: Baseline data and future research directions. Journal of Business Ethics, 73(4), 347-368.

CRUE (2012) Directrices para la introducción de la sostenibilidad en el currículum, http://www.crue.org/Documentos\%20compartidos/Declaraciones/Directrices_Sosteniblidad_Cru e2012.pdf

CRUE (2015) Institucionalización del Aprendizaje-Servicio como estrategia docente dentro del marco de la Responsabilidad Social Universitaria para la promoción de la Sostenibilidad en la Universidad,

http://www.crue.org/Documentos\%20compartidos/Recomendaciones\%20y\%20criterios\%20tecni cos/2.\%20APROBADA\%20INSTITUCIONALIZACION\%20ApS.pdf

Furco, A. (2009) Institutionalizing Service-Learning in Higher Education. University of Minnesota. Office for Public Engagement.

Furco, A. \& Billing, S.H. (eds.) (2002) Service-Learning: The Essence of the Pedagogy, Information Age Publishing, Greenwich.

Grau, F.X. (2014) The "Glocal" University, Artículo publicado en la web de la Global University Network for Innovation, http://www.guninetwork.org/articles/glocal-university

GUNi, Global University Network for Innovation (2017) Higher Education in the World 6. Towards a Socially Responsible University: Balancing the Global with the Local. Girona: GUNi.

Hartman, L. P., \& Werhane, P. H. (2009). A modular approach to business ethics integration: at the intersection of the stand-alone and the integrated approaches. Journal of Business Ethics, 90(3), 295-300.

iGR, Grupo de investigación Ingeniería y Gestión Responsable (2016) Responsabilidad social en las universidades: Del conocimiento a la acción. Forética.

Jabbour, C.J., Sarkis, J., Lopes de Sousa, A.B. \& Govindan, K. (2013) Understanding the process of Greening of Brazilian Business Schools, Journal of Cleaner Production, 61, 25-35. 
Larrán, M. y Andrades, F.J. (2013) El marco conceptual de la responsabilidad social universitaria. Granada: Foro de Consejos Sociales de las Universidades Públicas de Andalucía.

Lozano, R. (2006) Incorporation and institutionalization of SD into universities: breaking through barriers to change, Journal of Cleaner Production, 14 (9-11), 787-796.

Lozano, R., Ceulemans, K., Alonso-Almeida, M., Huisingh, D., Lozano, F. J., Waas, T., Lambrechts, W., Lukman, R., Hugé, J. (2014) A review of commitment and implementation of sustainable development in higher education: results from a worldwide survey. Journal of Cleaner Production 108, Part A, 1-18.

Marquès, M. (2014) La dimensión docente de la Responsabilidad Social Universitaria: la institucionalización del aprendizaje servicio en la Universitat Rovira i Virgili, Comunicación presentada a las I Jornadas Internacionales sobre Responsabilidad Social Universitaria Cádiz, 2021 de febrero de 2014.

Martínez, M. (ed.) (2010) Aprendizaje servicio y responsabilidad social de las universidades, ICE y Ediciones Octaedro, Barcelona.

Martínez, O. y Picco, J. E. (2001). Responsabilidad social de las Universidades. Buenos Aires: DT.UBA.

Ministerio de Educación (2011a) Estrategia Universidad 2015. Contribución de las universidades al progreso socioeconómico español 2010-2015. Ministerio de Educación; Secretaría General Técnica.

Ministerio de Educación (2011b) La responsabilidad social de la universidad y el desarrollo sostenible. Documento elaborado por la Comisión Técnica de la Estrategia Universidad 2015. Ministerio de Educación; Secretaría General Técnica.

Peix, E., Villà, R., Betts,A., Martínez, P. y Prat, J. (2017) "ESDU: Educación para el Desarrollo Sostenible en la Universidad", Comunicación presentada al VII Congreso Universidad y Cooperación al Desarrollo: La Universidad y los Objetivos de Desarrollo Sostenible, Madrid, 29-31 de marzo de 2017.

Rowe, D. (2007). Sustainability: Education for a sustainable future. Science, 317, 323-324.

Ruiz-Corbella, M. y Bautista-Cerro, M.J. (2016) La responsabilidad social en la universidad española, Teoría de la Educación 28(1): 159-188.

Ryan, A., Cotton, D. (2013) Times of change: Shifting pedagogy and curricula for future sustainability. In: The Sustainable University Progress and Prospects, Sterling, S., Maxey, L., Luna, H. (eds.), Abingdon, Routledge/Earthscan.

Setó-Pamies, D. \& Papaoikonomou, E. (2016) A multi-level perspective for the integration of ethics, corporate social responsibility and sustainability (ECSRS) in management education, Journal of Business Ethics, 136 (3): 523-538.

Sibbel, A. 2009. Pathways towards sustainability through higher education. International Journal of Sustainability in Higher Education, 10(1), 68-82. 
Sipos, Y., Bryce, B. y Kurt, G. (2008). Achieving transformative sustainability learning: Engaging head, hands and heart. International Journal of Sustainability in Higher Education, 9(1), 68-86.

Steinemann, A. (2003). Implementing sustainable development through problem-based learning: Pedagogy and practice. Journal of Professional Issues in Engineering Education and Practice, 129(4), 216-224.

Sutcliffe, H. (2011) A report on Responsible Research \& Innovation, Matter ((https://ec.europa.eu/research/science-society/document_library/pdf_06/rri-report-hilarysutcliffe_en.pdf)

URV (2017) Informe del Programa d'Aprenentatge Servei de la Universitat Rovira i Virgili 2016-17, http://www.urv.cat/media/upload/arxius/aprenentatgeservei/Docs\%20APS/Informe\%20APS\%202016.pdf

Villà, R. (dir.) (2015) L'educació per al desenvolupament a la universitat. Impulsant l'educació crítica i compromesa als estudis de grau. Diagnosi de l'estat actual d'implementació, possibilitats i estratègies en l'educació formal de les universitatspubliques catalanes. Fundació Autònoma Solidària, Bellaterra 
